\title{
Building a culture of early mobilization in the pediatric intensive care unit-a nuts and bolts approach
}

\author{
Brenda M. Morrow^ \\ Department of Paediatrics and Child Health, University of Cape Town, Cape Town, South Africa \\ Correspondence to: Brenda M. Morrow. Department of Paediatrics and Child Health, $5^{\text {th }}$ Floor ICH Building, Red Cross War Memorial Children's \\ Hospital, Klipfontein Rd, Rondebosch, 7700, Cape Town, South Africa. Email: Brenda.morrow@uct.ac.za.
}

\begin{abstract}
The culture of sedation and immobilization in the pediatric intensive care unit (PICU) is associated with PICU-acquired weakness, delirium, and poor functional, neurocognitive and psychosocial outcomes. A structured approach to introducing physical activity, as early as possible after PICU admission, may prevent these complications and optimize the holistic outcomes of critically ill children. Changing culture and introducing new clinical practice in PICU is complex, but can be approached systematically, using a "nuts and bolts" approach targeting the basic, practical considerations and essential required elements or components. Extending the construction analogy, this article reviews the relevant literature to describe the essential elements required to build and sustain a successful and safe early mobility program in the PICU. Effective early mobilization requires individual patient assessment and goal setting, using a collaborative inter-disciplinary, patient- and family-centered approach, to ensure mobility goals and physical activities are appropriate for the patient's age, condition/s, premorbid function, strength, endurance and developmental level. Early mobility activities for the pediatric age spectrum include active or active-assisted range of motion exercises, neurodevelopmental play, use of mobility devices, in-bed exercises, transfers, sitting or standing tolerance, crawling, pre-gait activities, ambulation and activities of daily living, with a focus on play as function. Although there are few complete contraindications to early mobilization, appropriate precautions and preparation should be taken to mitigate potential safety concerns. Although there are many perceived barriers to early mobilization in the PICU, at the level of patient, provider, institution and knowledge translation; these are not objectively associated with increased risk during mobilization and can be overcome through an engaged process of practice change by all members of the interprofessional clinical team. Early mobility programs could be initiated in PICU as systematic quality improvement initiatives, with established processes to optimize structural, process and system elements and to provide continual feedback, measurement, benchmarking and collaboration; to ultimately impact on measurable patient outcomes. Early, graded, and individually prescribed mobilization should be considered as part of the standard PICU "package of care" for all critically ill and injured children, in order to improve their functional status and quality of life after PICU discharge.
\end{abstract}

Keywords: Pediatric critical care; early mobilization; rehabilitation; functional outcomes; quality improvement

Submitted Oct 07, 2020. Accepted for publication Feb 26, 2021.

doi: $10.21037 /$ tp-20-324

View this article at: http://dx.doi.org/10.21037/tp-20-324

^ ORCID: 0000-0001-5193-918X. 


\section{The foundation}

"The very first requirement in a hospital is that it should do the sick no harm"-Florence Nightingale

All construction requires a firm foundation. In the context of building clinical practice, this foundation can be considered to be the underlying theory and evidence supporting the proposed clinical interventions.

The well-known phrase coined by Friedrich Nietzsche, "That which does not kill us makes us stronger", is unfortunately not true for pediatric critical illness. With improved knowledge and technology there has been a marked improvement in survival of children admitted to pediatric intensive care units (PICU) across the world (1). However, this improved mortality has been accompanied by an increased burden of physical, neurocognitive and psychosocial morbidities that potentially persists for years after PICU discharge (1-4), affecting the quality of life of both the surviving child and family (5-12). These sequelae of critical illness have been collectively termed "post-intensive care syndrome" (PICS).

Predisposing factors for PICS are likely to be multifactorial, including the critical illness itself, nutritional factors, delirium and prolonged bedrest and immobility during the period of illness (7,13-15). Immobility alone has been shown to affect multiple body functions, with deleterious effects on the musculoskeletal, skin, respiratory, cardiovascular, hematological, metabolic, neurological, renal and gastrointestinal systems (16-20).

Intensive care unit (ICU)-acquired weakness, including critical illness polyneuromyopathy (CIP), is well described in adults $(14,21)$ and has also been observed in children admitted to PICU, associated with immobility, sepsis and multi-organ dysfunction (22-25) and persisting for up to one year post discharge (25). Delirium has also been well described in both infants and children in PICU (26-28), associated with severity of illness, sedative over-use, sleep disruption, and prolonged bedrest with immobility $(14,29,30)$. Delirium itself places children at increased risk of mortality and morbidity, with longer duration of hospital stay, prolonged mechanical ventilation, and post-traumatic stress disorder after discharge (30). Immobility and deep sedation remain common practices in PICU (31,32), and it has been suggested that immobility may be the most modifiable factor in PICU contributing to functional disability (33).

Whilst the focus of PICU care is, by necessity, on saving lives (34), there should be a simultaneous focus on preventing modifiable factors contributing to complications such as delirium and CIP and ultimately to improve the holistic, functional outcomes of pediatric critical illness. Because functional outcomes and speed of recovery cannot be predicted on the basis of illness severity or predicted mortality scores on admission, every child admitted to PICU should be offered access to appropriate, interprofessional, graded rehabilitation from the time of admission (7). In adults, CIP has been shown to develop within the first 48 hours of ICU admission (21), therefore early intervention is considered necessary to prevent the neuromuscular sequelae of critical illness $(14,35,36)$.

Early mobilization (EM) is an essential part of ICU and PICU rehabilitation, variably defined in the pediatric context as the application of physical activity within the first 48 to 72 hours of PICU admission $(37,38)$ or, more pragmatically, initiated as soon as possible after admission, in the absence of contraindications (39).

In adults there is a growing body of evidence supporting EM in ICU from individual trials, although there are conflicting reports when results are synthesized in systematic reviews and meta-analyses. A Cochrane review of four randomized controlled trials $(n=690)$ concluded that there is still insufficient evidence to confidently support safety or efficacy for a number of outcome measures, including physical function, muscle strength and healthrelated quality of life (40). Similar results were reported by Okada et al. [2019] (41), in a systematic review of 11 studies with 1,322 participants, who were unable to show an advantage of EM versus usual care for mortality ( pooled relative risk 1.1; 95\% CI: $0.8-1.58$ ), duration of ICU stay (pooled mean difference $-1.54 ; 95 \% \mathrm{CI}:-3.3$ to 0.25 ), or health-related quality of life. This study did report superior muscle strength [using the Medical Research Council (MRC) grading Score] in participants receiving EM (mean difference 4.84; 95\% CI: 0.36-9.31), but other secondary functional and safety outcomes did not show superiority of either intervention or control group (41). Conversely, another recent systematic review, which included 23 trials $(n=2,308)$ concluded that EM does appear to decrease the incidence of ICU-acquired weakness (relative risk 0.6; $95 \%$ CI: 0.4-0.9) and increase the number of ventilator-free days (standardized mean difference 0.17 ; $95 \%$ CI: $0.02-0.31$ ) as well as the discharge rate (relative risk 1.16; $95 \%$ CI: 1.0 1.34) in critically ill adults (42). A subsequent systematic review also reported that EM in the ICU was associated with a decreased likelihood of developing ICU-acquired weakness (OR 0.63; 95\% CI: 0.43-0.92) (43). Synthesizing data and comparing results between trials is challenging considering the heterogeneity of population, intervention and outcome 
measurements used in different settings.

The benefits of EM in the PICU context are not yet supported by high-level evidence, as pediatric specific studies are still extremely limited $(12,14)$. A retrospective study of 600 children reported a longer duration of PICU stay and vaso-active infusions, as well as increased delirium in mobilized patients compared to those who were not mobilized (37), however the study design was not appropriate for the determination of efficacy or safety, and there was likely inherent selection bias, with sicker patients possibly being targeted for rehabilitation in clinical practice. A later systematic review of 11 studies (two pilot trials and nine observational studies) in 1,178 children suggested that EM in the PICU appears to be safe and feasible, however efficacy could not be determined for a number of outcomes including mortality, duration of PICU stay, and PICUacquired morbidity (12).

Small studies have thus far showed that progressive upper and lower limb mobilization using gaming consoles and/or cycle ergometry, as well as a range of passive or active movement-based activities focused on improving physical function and strength, are both safe and feasible $(12,14,39,44)$. The "PICU Up!" quality improvement initiative $(n=200)$ reported no associated adverse events with the use of a stratified early rehabilitation program (38). Similarly, recent point-prevalence (PARK-PICU) studies of mobilization practices in Europe and the USA reported only minor adverse events, most commonly transient changes in vital signs $(31,32)$. Studies in specialized populations, for example following placement of ventricular assist devices, liver transplantation, and patients on extracorporeal membrane oxygenation (ECMO) awaiting lung transplantation have also not described serious adverse events associated with mobilization activities (45-48). Comprehensive guidelines for the safe implementation of EM in PICU, based on expert consensus, have been published previously (15). Despite the growing interest in PICU EM, it appears that EM is still not standardly implemented. The European (EU) PARK-PICU study reported that $46 \%$ of children were mobilized out of bed by nurses, therapists, family members and others. Of concern was that $43 \%$ of patients never received a physical- or occupational therapy referral, despite a median length of PICU stay of 11 days, and a quarter of patients were not mobilized at all owing to actual or perceived contraindications and over-sedation (31). Similarly, in the United States (US) PARK-PICU sites, one fifth of patients were reported to be completely immobile (32).
This article builds on the above theoretical foundation, with the knowledge that excessive sedation and bedrest in PICU causes harm and the as-yet unproven assumption that incorporating EM into the holistic care of critically ill children is therefore beneficial. Rather than presenting a comprehensive review of academic theory, this narrative review, using a combination of available evidence from current literature, clinical experience and expert opinion, aims to describe the basic, practical considerations and essential elements (the "nuts and bolts") required to build and sustain a successful and safe EM program in the PICU.

\section{"How-to" guide to early mobilization}

"What patients want is not rocket science, which is really unfortunate because if it were rocket science, we would be doing it. We are great at rocket science. We love rocket science. What we're not good at are the things that are so simple and basic that we [often] overlook them". Laura Gilpin, Planetree Institute (bttps:// resources.planetree.org/homepage-6/).

Although definitions vary, for the purposes of this review, mobility interventions can be considered to include all physical activities aimed at preventing muscle wasting and preserving or enhancing functional mobility and cognitive recovery. Early mobilization refers to such activities implemented in the first few days after admission to PICU (after initial medical stabilization), although exact definitions and timepoints vary $(12,49,50)$. Early mobility and PICUbased rehabilitation prescription is not determined or supported by "rocket science" (there are not yet any rigorous clinical trials yielding high-level evidence), and therefore cannot be prescriptively and prohibitively defined. Without evidence to the contrary, good PICU mobilization practice can be summarized as following individualized, goaldirected planning to facilitate critically ill children to move as soon after PICU admission as appropriate, at a level and pace suitable to their age, condition and developmental level. Even where protocols have been implemented for research purposes, the majority have planned EM activities individually (49). Having unit-based mobility protocols are recommended, as these have been associated with improved mobility practice, and may reduce the potential for implicit bias in clinical decision making $(32,49)$.

\section{Setting the goals}

The general purpose of EM is to prevent critical illness- 
acquired morbidities such as muscle weakness and deconditioning; to optimize skin integrity, physical and neurocognitive health; to prevent PICU delirium; to reduce the duration of invasive mechanical ventilation and to enhance functional recovery (15). Whilst ambulation and independent mobilization out of bed are usually the mobility goals for adult rehabilitation, this may not be feasible or appropriate in children, considering the heterogenous age and developmental levels of patients admitted to PICU $(12,51)$. Considering the diversity of patients admitted to PICU, it is essential to develop mobilization goals on an individual basis for each patient. These goals should be appropriate to the child's age, specific condition, premorbid level of function, strength, endurance and developmental level (51).

The importance of interprofessional teamwork, discussion, and collaboration in setting individual patient mobility goals and implementing EM activities cannot be over-emphasized. Not least, a positive attitude, with motivation and support for EM from all staff involved in a patient's care, has been shown to comfort families and encourage patient participation in mobility activities (34).

\section{Suitable for all ages}

A pediatric study reported that EM was more strongly supported for older patients, with clinicians feeling that EM could be initiated in only $39 \%$ of the youngest invasively ventilated patients compared to $97 \%$ of the oldest patients (52). Similarly, studies from Canada and the USA have reported that increasing age is an independent predictor of EM $(32,37)$. However, typically developing infants spend a considerable number of hours per day moving (including voluntary and involuntary movement), and this movement is essential to ensure the attainment of normal developmental milestones. Furthermore, it has been reported that in the USA, two thirds of children admitted to PICU for more than 72 hours were under three years of age (32). It is therefore strongly suggested that young children and infants be specifically targeted to receive developmentally appropriate EM in PICU.

It is generally agreed that all patients admitted to the PICU, regardless of age and premorbid function, could benefit from PICU-based rehabilitation. Therefore all patients should be assessed for the appropriateness of EM, ideally within the first 24 hours of admission (15).

Early mobility activities for the pediatric age spectrum could include active or active-assisted range of motion exercises, neurodevelopmental play, use of a mobility device (e.g., cycle ergometer), in-bed mobility exercises, transfers (e.g., lying to sitting, bed to chair, sit to stand, active holding in caregivers arms), sitting or standing tolerance, crawling, pre-gait activities, ambulation and activities of daily living (15). Unconscious patients may still benefit from passive range of movement exercises (14). The type of activity chosen must be appropriate for the child's current and premorbid physical state, acuity of illness, age and developmental level $(12,53)$. This can only be determined through a thorough objective clinical assessment and subjective history from the parent/caregiver. The mobilization activity should be graded progressively though the child's PICU admission, as appropriate to their changing condition.

One of the main goals of EM is to facilitate independent function. In young children, the main functional activity of daily living is play, with the type of play changing as the child grows older and develops physically and neurocognitively. Play is essential for the development of physical motor, communication, and problem-solving skills, as well as facilitating emotional resilience and creativity (54), and importantly is also simply enjoyable. Children with life-threatening conditions are often unable to spontaneously engage in play, and this may have a longterm impact on function, including the acquisition of normal neurodevelopmental milestones (54).

Play provides cognitive stimulation as well as physically enabling a range of mobility activities and can be incorporated into PICU EM using both active and active assisted methods, with the type of play activity chosen according to individually developed EM goals. Using play in EM has numerous potential physical and emotional benefits, not least of which is the likelihood of improving patient and family buy-in and cooperation, leading to more effective therapy. This suggestion is supported by a quote from a qualitative study_- "be's a little guy, he doesn't want to just march on the spot for no reason" (34). Incorporating play into therapy may improve the experience the child has whilst in PICU (34), thereby potentially reducing the psychological sequelae of PICU admission. Examples of play activities include construction, for example using building blocks; active object play (e.g., throwing a ball or balloon); movement play, for example using interactive technologies with elements of virtual reality (e.g., WeeFit active games); and imaginative or pretend play (54). In a South African PICU we have even filled small paddling pools with warm water, so that critically ill children can safely engage in facilitated water play, even whilst invasively 
mechanically ventilated. We have had no adverse events with these types of activities, and have had excellent buy-in from patients, staff and families (unpublished data).

Before implementing a mobilization intervention, individualized mobilization/activity goals should be determined, including the child and family wherever possible. Prior to initiating a mobility activity with a patient, it should be ensured that sufficient personnel are available; that the environment is safe with no obstructions or other hazards; that airways and lines are well secured and wound dressings are intact; and the patient's pain and comfort must be optimized (15), through pharmacological and nonpharmacological methods.

\section{Dosage}

There is no data clearly supporting an optimal dose and/ or frequency of mobilization in PICU, but it has been recommended that one should aim for approximately 30 minutes of activity, once daily initially, progressing according to patient response and tolerance by increasing the level of mobilization; combining mobilization activities; and/or increasing the number and range of activities per day (15). In the absence of objective evidence, the optimal dose and frequency of EM activities should be determined according to individual patient presentation. For clinical research purposes it is important to standardize the progressive dose and frequency to the extent possible, in order to improve scientific rigor and reproducibility of the research.

\section{Warnings}

Contraindications to mobility interventions include severe hemodynamic, respiratory or neurological instability and surgical complications such as uncontrolled bleeding, coagulopathy, and unstable fractures. The presence of vaso-active infusions; invasive or non-invasive mechanical ventilation; external ventricular drainage; continuous renal replacement therapy; known or suspected deep vein thrombosis and confusion or delirium do not constitute contra-indications to mobilization, however for all critically ill children, appropriate precautions and preparation should be made to mitigate any potential safety concerns (15).

Mobilization attempts should be halted in the case of persistent desaturation; significant changes in vital signs; new or increasing signs of respiratory distress; concerns about airway, device or wound integrity; ventilatory asynchrony; marked increases in intracranial pressure; bleeding; change in mental status and behavior which places the child at risk of adverse events; or patient injury (15).

\section{Equipment}

A number of technological devices have been developed to assist with mobility activities in the ICU and PICU, including virtual reality games (e.g., Wii-Fit); tilt tables; custom built walkers and seating devices; neuromuscular electrical nerve stimulation; and cycle ergometers $(14,35,39,44,55)$. Many of these devices are, however, associated with substantial financial cost and are not globally available. Furthermore, there is no evidence that expensive devices are required for effective PICU-based rehabilitation, despite the lack of pediatric-specific mobility equipment being perceived as a barrier to implementation of EM (51). In my experience working in resource-constrained environments, most readily available, simple equipment and furniture can be adapted to patient requirements during mobilization.

In my opinion, the only absolute requirement for a successful EM initiative is the presence of a willing, dedicated and capable interprofessional and interdisciplinary PICU team. Early mobilization cannot be any one person's responsibility - to be successful, there must be buyin from the extended team, including nurses, intensive care specialists, respiratory technologists/technicians, rehabilitation therapists (e.g., physical, speech and occupational therapists) and most importantly-the child and family (14).

\section{The bigger picture}

"A single twig breaks, but the bundle of twigs is strong"Tecumseh

Early mobilization should not be conducted in isolation, but rather as part of a holistic, bundled approach. As such, $\mathrm{EM}$ is an important part of the $A B C D E F$ bundle of care, which includes: Assessment, Prevention, and Management of Pain, Both Spontaneous Awakening and Spontaneous Breathing Trials), Choice of analgesia and sedation, Delirium: Assess, Prevent, and Manage, Early mobility and Exercise, and Family engagement and empowerment $(56,57)$.

The ABDCEF bundle aims to facilitate earlier liberation from mechanical ventilation, reduce duration of PICU and hospital stay, improve survival and optimize patient functional outcomes $(56,58,59)$. This bundle has been shown to be safe, with a reported reduction in the incidence 
of delirium and an increased number of ventilator-free days after implementation (58). Although there is no clear evidence supporting the effectiveness of the bundle as a whole, each component of the bundle is evidence-based $(56,57)$, and for each component to be optimally effective, the other component bundle practices also need to be adhered to.

$A$-Assessment, Prevention, and Management of Pain: for EM to be successfully implemented and acceptable to the patient and family, it should not cause unnecessary pain or discomfort. It is therefore essential that pain is well managed and analgesia is optimized, without excessive sedation (29). It is suggested that pre-emptive analgesia be administered prior to mobility activities, where there is the possibility of causing pain. Older children may benefit from patient-controlled analgesia devices, to regulate their own pain control.

$B$-Both spontaneous awakening and spontaneous breathing trials: the combination of "sedation vacations", which is considered good practice in adult and pediatric ICUs, with rehabilitation sessions may provide an opportunity to implement mobility interventions with adequate patient cooperation and awareness $(29,36)$.

$C$ - Choice of analgesia and sedation: titrating sedation and analgesia to allow children to be more awake and alert during the day with normal, restorative sleep at night, facilitates their ability to participate in mobilization activities, whilst also reducing their risk of developing delirium (29). There is a tension between over-sedation, which can lead to longer duration of mechanical ventilation, delirium, prolonged immobility and increased healthcare costs; and undersedation, which can cause non-pain related distress and anxiety (potentially predisposing to post-traumatic stress disorder), accidental extubation and dislodgement of invasive lines and devices (60). Patient-specific and goal-directed sedation plans are essential to optimizing sedation and analgesia and creating a culture of EM in PICU. Monitoring sedation level through the use of validated scoring systems is considered good practice to formulate sedation goals and titrate drug doses, yet is inconsistently adhered to in many PICUs (29). Sedation scores that have been validated for use in the PICU include the COMFORT scale and the State Behavioral Scale $(29,60)$. Two systematic reviews have suggested that the COMFORT Scale has the greatest clinical utility in assessing pain, non-pain related distress and sedation in mechanically ventilated patients in the PICU $(60,61)$. The FLACC (Facial expression, Limbs, Cry, Consolability) scale for infants and children and the Multidimensional
Assessment Pain Scale (MAPS) are also recommended for the assessment of pain in PICU (60). If benzodiazepines are clinically necessary, the child should still be assessed for passive activity and positioning as mobility interventions (29).

$D$-Delirium assessment, prevention and management: delirium impacts on the ability of children to participate in physical activity, and has important clinical sequelae including prolonged mechanical ventilation and longterm psychosocial morbidity (30). Prolonged immobility and deep sedation (especially with benzodiazepines) are independent predictors for delirium, emphasizing the importance of a multi-modal approach to prevention and management, including sedation optimization and EM. Delirium screening and assessment are important quality control indicators and outcome measures for quality improvement initiatives, but this is generally poorly performed in the PICU $(29,30)$. The pediatric Confusion Assessment Method for ICU (pCAM-ICU), suitable for children from 5 years of age and the Cornell Assessment Pediatric Delirium tool (CAP-D), for children from birth, have been shown to be valid and reliable in the PICU, with the clinical utility established at the bedside (60). The CAP-D is the recommended instrument to assess pediatric delirium, based on a level A grade of recommendation (60).

E-Early mobilization.

F-Family engagement: the EU PARK-PICU study showed that family presence was the factor most strongly positively associated with out-of-bed mobilization, with an adjusted odds ratio of 7.8 (95\% CI: 3.1-19.8) (31). The equivalent PARK-PICU conducted in the United States also showed family presence was positively associated with out of bed mobility, but this was only apparent for young children under three years of age (adjusted odds ratio 4.55; 95\% CI: 3.1-6.6) (32).

In the spirit of patient- and family centered care, actively including the family in their child's rehabilitation process is invaluable (62), with qualitative reports of family support for EM as an important component of PICU care (34). It has been shown that family participation in the care of their critically ill child reduces parental anxiety and improves their satisfaction levels (63). A collaborative process is required to ensure that parents understand the rationale, benefits, and process of EM, whilst assuring them that appropriate safety measures will be implemented (34). Parents are best placed to determine their child's level of comfort and pain, and are best able to communicate (including non-verbal communication) with their young child (51), thereby improving the child's level of cooperation and motivation. For severely ill and 
highly sedated patients, as well as young infants, facilitating holding in the parents' arms is therapeutic for both child and parent. Positioning in this way, while not being truly 'active' mobilization, allows for natural movement and anti-gravity positioning, which is likely to be beneficial to the child. It has also been suggested that active family involvement allows some mobilization activities to be sustained beyond designated therapy sessions (34).

\section{Building a PICU quality culture}

"Never lose an opportunity of urging a practical beginning, however small, for it is wonderful how often in such matters the mustard-seed germinates and roots itself."-Florence Nightingale

Many perceived barriers to EM in the PICU have been reported in different studies, at the level of patient, provider, institution and knowledge translation $(34,51)$. Resource and time constraints and increased workload are commonly cited; safety concerns are common, with patients often perceived as being "too sick" and physiologically unstable to safely tolerate mobilization, especially early in their admission; sedation practices may limit the cooperative ability of patients; the patients themselves may lack motivation or the ability to cooperate; the presence of invasive lines, airways and devices create concern about dislodging during movement; certain medications may be associated with increased risk; and delirium may further limit patient participation (29,31,32,34,35,52,64-67). Owing to these perceptions of risk, the youngest and sickest patients tend to receive the fewest rehabilitation interventions, and likely remain at the greatest risk of PICS (67). However, these perceived barriers have not been objectively shown to be associated with increased risk during mobilization $(12,31,32,35,68)$.

Early mobility programs can be developed as practice/ quality improvement (QI) initiatives in PICU, either independently or as part of an ABCDEF bundle, using locally developed and/or established protocols. Despite knowing what the "nuts and bolts" of EM are, there are marked challenges in implementing the necessary strategies to ensure consistent, sustained and effective implementation. Developing interprofessional teams to plan and implement EM may require cultural shifts and organizational changes, but has been shown to be effective in increasing the proportion of patients receiving EM, as well as reducing the time to mobilize (12).

Culture change is an important component of any QI initiative, and is essential to facilitate EM of critically ill children (51). Changing culture, however, is not easy and it is essential to include all stakeholders in the process, with education, discussion and brainstorming to develop a common vision and commitment to work together for improved functional patient outcomes (50). Attention must first and foremost be paid to changing the attitudes of PICU leaders (intensivists; nurse managers; administrators) to support and champion for rehabilitation programs in their units (69).

Quality healthcare delivery requires integrated elements of structure (e.g., people/staffing, equipment, education, data collection), process (e.g., policies, protocols, procedures), and systems (e.g., programs, organization, culture, leadership) that interplay through continual feedback, measurement, benchmarking and collaboration, to ultimately impact on patient outcomes (e.g., patient safety, quality, satisfaction) (Figure 1A) (70,71).

Evaluating local PICU practice and potential or perceived barriers to EM is a good first step to initiating change in practice, by identifying modifiable structural, process and system factors which can be focused areas of practice improvement (71) (Figure 1B). This can also help identify the specific problems and practice gaps to clarify the goal/s of the practice improvement initiative, the first required step for culture change $(51,72)$. For example, Herbsmann et al. [2020] performed a "gap analysis" which highlighted the limited mobilization of patients in their PICU, as well as perceived and actual barriers to EM. As a result of this preliminary analysis, a targeted QI project was implemented aimed primarily at increasing the proportion of children receiving EM and secondarily to improving patient clinical outcomes (PICU length of stay and proportion discharged home) (73). Wieczorek et al. [2016] implemented the "PICU UP!" QI initiative, initially conducting focus groups with all relevant stakeholders to present the problem and identify both facilitators and barriers to EM in the PICU (38).

The necessary staff, procedures, policies, and equipment for the QI initiative should be identified and mobilized, with sufficient interaction, collaboration and knowledge sharing to create a common sense of purpose and urgency, with buy-in from all important stakeholders (51). Provision of effective leadership and consistent, interprofessional, staffing for the program, for example by establishing EM "champions", has been shown to improve implementation and sustainability of QI initiatives $(51,71)$. In addition, provision of interprofessional training, education and 
A

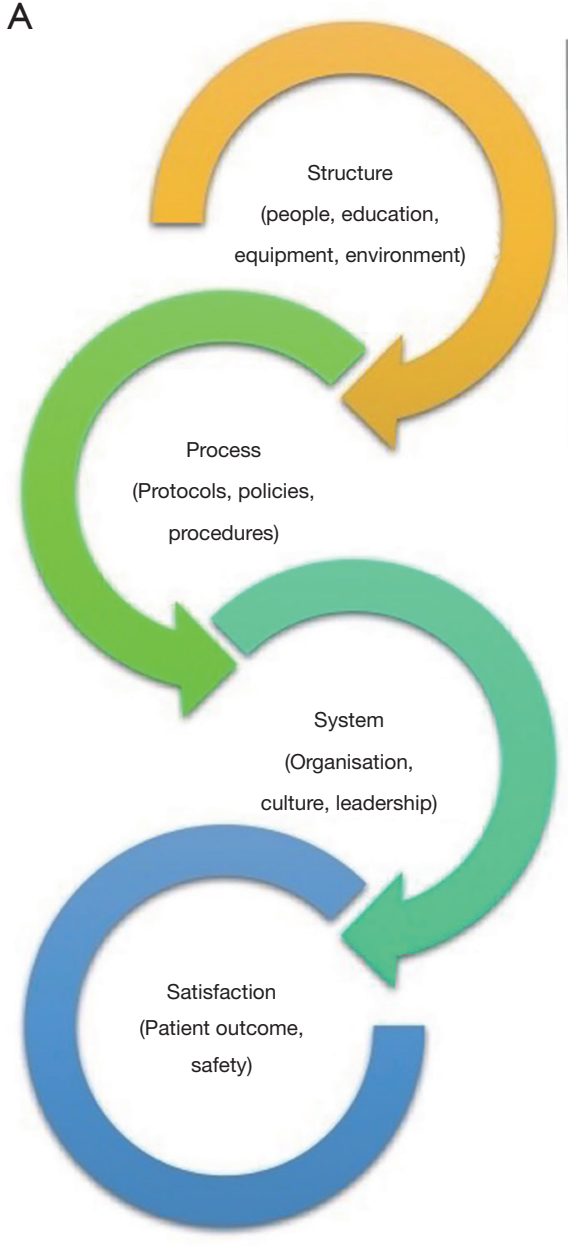

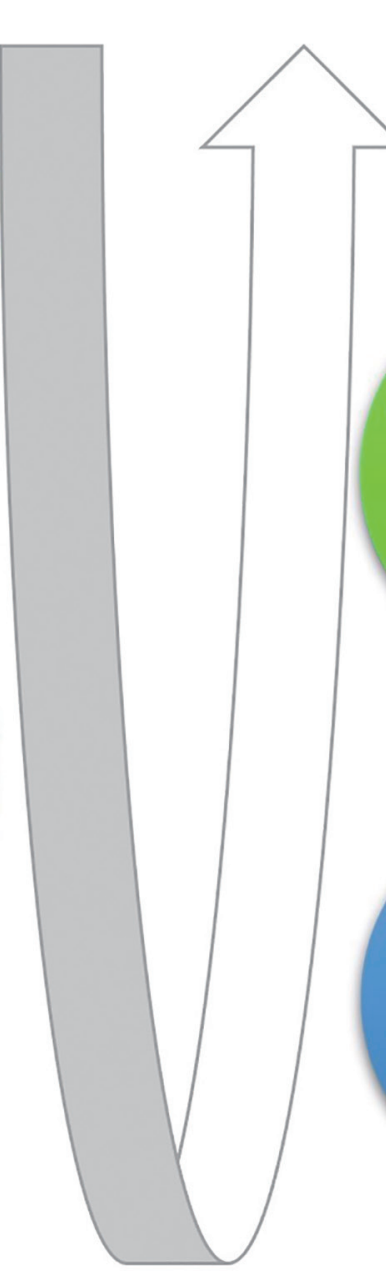

B

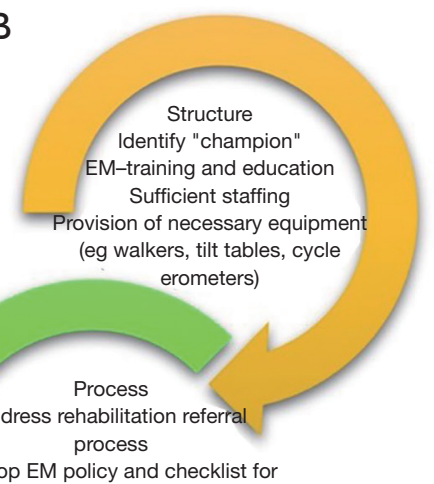

evelop EM policy and checklist for

PICU

Identify and address perceived

System Mobilise institutional leadership Prioritise budget Promote institutional culture

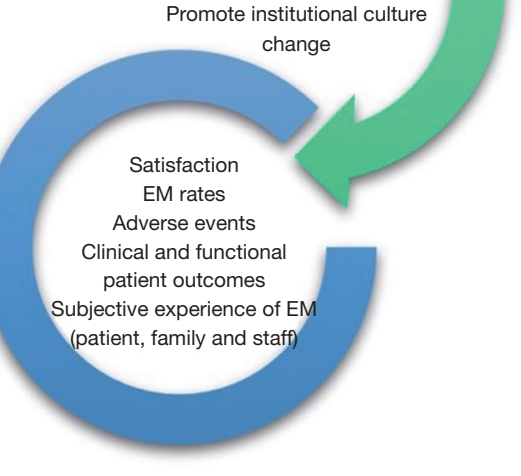

Figure 1 Essential components of quality improvement initiatives. (A) General principles (70); (B) principles applied to Early Mobilisation program development and implementation.

support leads to better uptake and faster implementation of practice change (74). Considering that multiple professionals contribute to EM activities (55), the professional category of the EM "champion" may not be important. Both the EU and USA PARK-PICU studies, for example, showed that nurses provided the majority of mobilization events $(31,32)$, whilst in another study rehabilitation was most commonly provided by physical therapists (37). Wieczorek [2016] used "champions" from each of the professional groups contributing to the PICU interprofessional team from the outset of their project (38).

In their QI project, Herbsman et al. [2020] mobilized family/patient-centered care team to improve a number of process measures related to EM, with interventions to increase rehabilitation referrals and activity orders; remove barriers to mobilization; educating and training multiple stakeholders on the benefits and methods to safely mobilize patients; develop an algorithm to identify patients appropriate for mobilization and ultimately change the culture of EM within the PICU (73). The interprofessional team of "champions" in the "PICU-Up!" program developed guidelines for EM using feedback from their initial focus groups as well as existing literature. Educational resources were also developed for all PICU staff (38).

An example of a specific process that could be targeted for change is the requirement, in many PICUs, for a physician referral or order before physical therapy (and hence early mobilization) can be initiated $(14,32)$. This could result in unacceptable delays before patient assessment and implementing mobility activities. It has been 
suggested that making EM standard of care for all patients unless specifically ordered otherwise, could address this concern (46,75). One of the outcomes of interest related to this process change could be the time from admission to first physical activity.

Establishing measurement and benchmarking processes and systems is essential early on in the QI process, in order to determine if the practice change is being successfully implemented; if implementation is sustained; and whether the interventions actually translate to improved patient, family and staff satisfaction and/or patient outcomes (71). Without baseline data, any improvements will not be detectable. Measures should ideally consider structural, process and clinical outcomes (71). For example, measurements could include reporting the number of patients mobilized/patient bed days over a set time period, or "missed opportunities" for EM (process measures of adherence); availability of equipment or appropriate rehabilitation staff (structural measure); and appropriate, feasible, valid and reliable measures of clinical outcome (71) (e.g., incidence of delirium; duration of mechanical ventilation and PICU stay; patient/ family satisfaction; functional outcomes). Data collection methods should ideally be integrated into the daily workflows of clinical staff, to ensure sustainability and accuracy (71).

Herbsmann et al. [2020], for example, documented their primary QI outcome as the percentage of patients mobilized early, according to their stated definition, whilst process measures included the percentage of patients with therapy and activity orders as well as perceived barriers to EM. Secondary outcome measures included any adverse events, PICU and hospital length of stay and discharge disposition (73). The "PICU-Up!" program initiated a process of setting activity levels and activity goals for each patient during the bedside round, which was documented in the daily records (38). Primary outcomes were the proportion of physical and/or occupational therapy referrals by day 3 of PICU admission and the number and type of mobilization activity performed by day 3 ). Secondary outcomes were the number of activities that were stopped (with reasons), barriers to activity, and mobilization-related adverse events. These measures were collected every day of PICU admission for all patients, but for reporting purposes averages and counts over a pre- and post-intervention periods were compared (38).

It would be ideal to measure muscle strength and function as clinically relevant outcomes of an EM QI program. Objective assessment of muscle strength is however challenging in the pediatric population, with both the
MRC grade score and dynamometry tools being volitional measures requiring patient cooperation, understanding and motivation. They are therefore not reliable or feasible in young children or infants, or for patients receiving high levels of sedative or analgesic infusions (14). For clinical practice, a gross indication of strength can be made by observing spontaneous, assisted and/or resisted movement efforts, however this would not be an appropriate outcome measure for clinical or QI research reporting. The following validated scores of function have been suggested for use as PICU outcome measures: the Functional Status Scale (FSS), the Pediatric Cerebral Performance Category (PCPC) and the Pediatric Overall Performance Category (POPC). The FSS has been shown to be reliable, rapid, is applicable to a broad age spectrum, and is sufficiently sensitive to demonstrate changed functional ability from pre- to post hospital admission (76). Furthermore, the FSS is more objective, precise and granular than the POPC/ PCPC methods (77), and may therefore be preferable for use in PICU. A pediatric version of the adult validated Chelsea Critical Care Physical Assessment Tool (CPAx) (78) has been developed through a modified Delphi process (79), but clinical validation of this tool in the PICU context is still pending.

Particularly in resource-constrained settings, staff shortages may be perceived to limit the ability to mobilize patients effectively, as a coordinated team approach is optimal. However, implementing "mobility teams" has not been associated with increased costs in other settings; and if PICU length of stay is shortened as a result of the intervention, it is likely that there will be associated cost savings (80-82). A cost-effectiveness analysis might therefore be an important indicator to feed back to hospital managers to encourage the long-term sustaining of EM quality improvement projects.

Quality improvement measurements need to be audited regularly, fed back to stakeholders, and analyzed to identify further modifiable factors to fine-tune and continually improve the program (71). This process could be implemented using a "Plan, Do, Study, Act" (PDSA) model of practice improvement (73), which ensures the EM program is feasible, acceptable to staff and families, effective, and appropriately responsive to the specific and changing dynamic within different PICUs. Herbsman et al. [2020] used a process control chart to track and report the proportion of patients mobilized over time (marking intervention points), with weekly reporting periods; whilst perceived barriers (process measures) and secondary outcome measures were 
reported pre- and post-intervention (73).

\section{Conclusions}

"We are what we repeatedly do. Excellence, then, is not an act, but a babit." - Aristotle

Early mobilization should be considered as part of the standard PICU "package of care" for all critically ill and injured children. Most perceived barriers to EM are surmountable and can be overcome through an engaged process of practice change by all members of the interprofessional clinical team.

By offering graded rehabilitation, appropriate to the child's condition and acuity of illness, we can be responsive to the needs of the critically ill child, and ultimately improve their functional status at and after PICU discharge. Through the combined efforts of the interprofessional team, it is possible to build a PICU culture that supports EM and other best care practices, thus ensuring that more children are able to return to their homes, families and communities after PICU admission, alive and able to live their lives to the fullest.

\section{Acknowledgments}

Funding: None.

\section{Footnote}

Provenance and Peer Review: This article was commissioned by the Guest Editors (Jan Hau Lee, Vijay Srinivasan, and Debbie Long) for the series "Pediatric Critical Care" published in Translational Pediatrics. The article has undergone external peer review.

Conflicts of Interest: The author has completed the ICMJE uniform disclosure form (available at http://dx.doi. org/10.21037/tp-20-324). The series "Pediatric Critical Care" was commissioned by the editorial office without any funding or sponsorship. The author has no conflicts of interest to declare.

Ethical Statement: The author is accountable for all aspects of the work in ensuring that questions related to the accuracy or integrity of any part of the work are appropriately investigated and resolved.

Open Access Statement: This is an Open Access article distributed in accordance with the Creative Commons Attribution-NonCommercial-NoDerivs 4.0 International License (CC BY-NC-ND 4.0), which permits the noncommercial replication and distribution of the article with the strict proviso that no changes or edits are made and the original work is properly cited (including links to both the formal publication through the relevant DOI and the license). See: https://creativecommons.org/licenses/by-nc-nd/4.0/.

\section{References}

1. Namachivayam P, Shann F, Shekerdemian L, et al. Three decades of pediatric intensive care: Who was admitted, what happened in intensive care, and what happened afterward. Pediatr Crit Care Med 2010;11:549-55.

2. Pollack MM, Holubkov R, Funai T, et al. Pediatric intensive care outcomes: development of new morbidities during pediatric critical care. Pediatr Crit Care Med 2014;15:821-7.

3. Bone MF, Feinglass JM, Goodman DM. Risk factors for acquiring functional and cognitive disabilities during admission to a PICU*. Pediatr Crit Care Med 2014;15:640-8.

4. Farris RW, Weiss NS, Zimmerman JJ. Functional outcomes in pediatric severe sepsis: further analysis of the researching severe sepsis and organ dysfunction in children: a global perspective trial. Pediatr Crit Care Med 2013;14:835-42.

5. Ambuehl J, Karrer A, Meer A, et al. Quality of life of survivors of paediatric intensive care. Swiss Med Wkly 2007;137:312-6.

6. Jayshree M, Singhi SC, Malhi P. Follow up of survival and quality of life in children after intensive care. Indian Pediatrics 2003;40:303-9.

7. Taylor A, Butt W, Ciardulli M. The functional outcome and quality of life of children after admission to an intensive care unit. Intensive Care Med 2003;29:795-800.

8. Knoester H, Bronner MB, Bos AP. Surviving pediatric intensive care: physical outcome after 3 months. Intensive Care Med 2008;34:1076-82.

9. Knoester H, Bronner MB, Bos AP, et al. Quality of life in children three and nine months after discharge from a paediatric intensive care unit: a prospective cohort study. Health Qual Life Outcomes 2008;6:21-7525-6-21.

10. Als LC, Nadel S, Cooper M, et al. Neuropsychologic function three to six months following admission to the PICU with meningoencephalitis, sepsis, and other disorders: a prospective study of school-aged children. Crit 
Care Med 2013;41:1094-103.

11. Als LC, Tennant A, Nadel S, et al. Persistence of Neuropsychological Deficits Following Pediatric Critical Illness. Crit Care Med 2015;43:e312-5.

12. Cuello-Garcia CA, Mai SHC, Simpson R, et al. Early Mobilization in Critically Ill Children: A Systematic Review. J Pediatr 2018;203:25-33.e6.

13. Hermans G, Van den Berghe G. Clinical review: intensive care unit acquired weakness. Crit Care 2015;19:274.

14. Cameron S, Ball I, Cepinskas G, et al. Early mobilization in the critical care unit: A review of adult and pediatric literature. J Crit Care 2015;30:664-72.

15. Choong K, Canci F, Clark H, et al. Practice recommendations for early mobilisation in critically ill children. J Pediatr Intensive Care 2018;7:14-26.

16. Knight J, Nigam Y, Jones A. Effects of bedrest 2: gastrointestinal, endocrine, renal, reproductive and nervous systems. Nurs Times 2009;105:24-7.

17. Knight J, Nigam Y, Jones A. Effects of bedrest 1: cardiovascular, respiratory and haematological systems. Nurs Times 2009;105:16-20.

18. Nigam Y, Knight J, Jones A. Effects of bedrest 3: musculoskeletal and immune systems, skin and selfperception. Nurs Times 2009;105:18-22.

19. Pavy-Le Traon A, Heer M, Narici MV, et al. From space to Earth: advances in human physiology from 20 years of bed rest studies (1986-2006). Eur J Appl Physiol 2007;101:143-94.

20. Brower RG. Consequences of bed rest. Critical Care Medicine 2009;37:S422-8.

21. Schweickert WD, Hall J. ICU-acquired weakness. Chest 2007;131:1541-9.

22. Choong K, Al-Harbi S, Siu K, et al. Functional recovery following critical illness in children: the "wee-cover" pilot study. Pediatr Crit Care Med 2015;16:310-8.

23. Petersen B, Schneider C, Strassburg HM, Schrod L. Critical illness neuropathy in pediatric intensive care patients. Pediatr Neurol 1999;21:749-53.

24. Williams S, Horrocks IA, Ouvrier RA, et al. Critical illness polyneuropathy and myopathy in pediatric intensive care: A review. Pediatr Crit Care Med 2007;8:18-22.

25. Banwell BL, Mildner RJ, Hassall AC, et al. Muscle weakness in critically ill children. Neurology 2003;61:1779-82.

26. Smith HA, Fuchs DC, Pandharipande PP, et al. Delirium: an emerging frontier in the management of critically ill children. Crit Care Clin 2009;25:593-614, x.

27. Silver GH, Kearney JA, Kutko MC, et al. Infant delirium in pediatric critical care settings. Am J Psychiatry 2010;167:1172-7.

28. Smeets IA, Tan EY, Vossen HG, et al. Prolonged stay at the paediatric intensive care unit associated with paediatric delirium. Eur Child Adolesc Psychiatry 2010;19:389-93.

29. Saliski M, Kudchadkar SR. Optimising sedation management to promote early mobilisation for critically ill children. J Pediatr Intensive Care 2015;4:188-93.

30. Norman S, Taha AA, Turner HN. Delirium in the Critically Ill Child. Clin Nurse Spec 2017;31:276-84.

31. Ista E, Scholefield BR, Manning JC, et al. Mobilization practices in critically ill children: a European point prevalence study (EU PARK-PICU). Crit Care 2020;24:368.

32. Kudchadkar SR, Nelliot A, Awojoodu R, et al. Physical Rehabilitation in Critically Ill Children: A Multicenter Point Prevalence Study in the United States. Crit Care Med 2020;48:634-44.

33. Tabarki B, Coffinieres A, Van Den Bergh P, et al. Critical illness neuromuscular disease: clinical, electrophysiological, and prognostic aspects. Arch Dis Child 2002;86:103-7.

34. Zheng K, Sarti A, Boles S, et al. Impressions of Early Mobilization of Critically Ill Children-Clinician, Patient, and Family Perspectives. Pediatr Crit Care Med 2018;19:e350-e357.

35. Lipshutz AK, Gropper MA. Acquired neuromuscular weakness and early mobilization in the intensive care unit. Anesthesiology 2013;118:202-15.

36. Schweickert WD, Pohlman MC, Pohlman AS, et al. Early physical and occupational therapy in mechanically ventilated, critically ill patients: a randomised controlled trial. Lancet 2009;373:1874-82.

37. Choong K, Foster G, Fraser DD, et al. Acute rehabilitation practices in critically ill children: a multicenter study. Pediatr Crit Care Med 2014;15:e270-9.

38. Wieczorek B, Ascenzi J, Kim Y, et al. PICU Up!: Impact of a Quality Improvement Intervention to Promote Early Mobilization in Critically Ill Children. Pediatr Crit Care Med 2016;17:e559-e566.

39. Choong K, Awladthani S, Khawaji A, et al. Early Exercise in Critically Ill Youth and Children, a Preliminary Evaluation: The wEECYCLE Pilot Trial. Pediatr Crit Care Med 2017;18:e546-e554.

40. Doiron KA, Hoffmann TC, Beller EM. Early intervention (mobilization or active exercise) for critically ill adults in the intensive care unit. Cochrane Database Syst Rev 2018;3:CD010754.

41. Okada Y, Unoki T, Matsuishi Y, et al. Early versus delayed 
mobilization for in-hospital mortality and health-related quality of life among critically ill patients: a systematic review and meta-analysis. J Intensive Care 2019;7:57.

42. Zhang L, Hu W, Cai Z, et al. Early mobilization of critically ill patients in the intensive care unit: A systematic review and meta-analysis. PLoS One 2019;14:e0223185.

43. Anekwe DE, Biswas S, Bussieres A, et al. Early rehabilitation reduces the likelihood of developing intensive care unit-acquired weakness: a systematic review and meta-analysis. Physiotherapy 2020;107:1-10.

44. Abdulsatar F, Walker RG, Timmons BW, et al. "Wii-Hab" in critically ill children: a pilot trial. J Pediatr Rehabil Med 2013;6:193-204.

45. Hollander SA, Hollander AJ, Rizzuto S, et al. An inpatient rehabilitation program utilizing standardized care pathways after paracorporeal ventricular assist device placement in children. J Heart Lung Transplant 2014;33:587-92.

46. Tsuboi N, Nozaki H, Ishida Y, et al. Early mobilization after pediatric liver transplantation. J Pediatr Intensive Care 2017;6:199-205.

47. Rehder KJ, Turner DA, Hartwig MG, et al. Active rehabilitation during extracorporeal membrane oxygenation as a bridge to lung transplantation. Respir Care 2013;58:1291-8.

48. Turner DA, Cheifetz IM, Rehder KJ, et al. Active rehabilitation and physical therapy during extracorporeal membrane oxygenation while awaiting lung transplantation: a practical approach. Crit Care Med 2011;39:2593-8.

49. Piva TC, Ferrari RS, Schaan CW. Early mobilization protocols for critically ill pediatric patients: systematic review. Rev Bras Ter Intensiva 2019;31:248-57.

50. Walker TC, Kudchadkar SR. Early mobilization in the pediatric intensive care unit. Transl Pediatr 2018;7:308-13.

51. Hopkins RO, Choong K, Zebuhr CA, et al. Transforming PICU culture to facilitate early rehabilitation. J Pediatr Intensive Care 2015;4:204-11.

52. Joyce CL, Taipe C, Sobin B, et al. Provider Beliefs Regarding Early Mobilization in the Pediatric Intensive Care Unit. J Pediatr Nurs 2018;38:15-9.

53. Wieczorek B, Burke C, Al-Harbi A, et al. Early mobilization in the pediatric intensive care unit: a systematic review. J Pediatr Intensive Care 2015;2015:129-70.

54. Nijhof SL, Vinkers CH, van Geelen SM, et al. Healthy play, better coping: The importance of play for the development of children in health and disease. Neurosci Biobehav Rev 2018;95:421-9.

55. Parchem K, Peck A, Tales K. A Multidisciplinary Approach to Equipment Use in Pediatric Patient Mobilization. Crit Care Nurs Q 2018;41:330-9.

56. Ely EW. The ABCDEF Bundle: Science and Philosophy of How ICU Liberation Serves Patients and Families. Crit Care Med 2017;45:321-30.

57. Marra A, Ely EW, Pandharipande PP, et al. The ABCDEF Bundle in Critical Care. Crit Care Clin 2017;33:225-43.

58. Balas MC, Vasilevskis EE, Olsen KM, et al. Effectiveness and safety of the awakening and breathing coordination, delirium monitoring/management, and early exercise/ mobility bundle. Crit Care Med 2014;42:1024-36.

59. Morandi A, Brummel NE, Ely EW. Sedation, delirium and mechanical ventilation: the 'ABCDE' approach. Curr Opin Crit Care 2011;17:43-9.

60. Harris J, Ramelet AS, van Dijk M, et al. Clinical recommendations for pain, sedation, withdrawal and delirium assessment in critically ill infants and children: an ESPNIC position statement for healthcare professionals. Intensive Care Med 2016;42:972-86.

61. Dorfman TL, Sumamo Schellenberg E, Rempel GR, et al. An evaluation of instruments for scoring physiological and behavioral cues of pain, non-pain related distress, and adequacy of analgesia and sedation in pediatric mechanically ventilated patients: A systematic review. Int J Nurs Stud 2014;51:654-76.

62. Davidson JE, Aslakson RA, Long AC, et al. Guidelines for Family-Centered Care in the Neonatal, Pediatric, and Adult ICU. Crit Care Med 2017;45:103-28.

63. Bastani F, Abadi TA, Haghani H. Effect of Family-centered Care on Improving Parental Satisfaction and Reducing Readmission among Premature Infants: A Randomized Controlled Trial. J Clin Diagn Res 2015;9:SC04-8.

64. Bailey P, Thomsen GE, Spuhler VJ, et al. Early activity is feasible and safe in respiratory failure patients. Crit Care Med 2007;35:139-45.

65. Stiller K. Safety issues that should be considered when mobilizing critically ill patients. Crit Care Clin 2007;23:35-53.

66. Choong K, Koo KK, Clark H, et al. Early mobilization in critically ill children: a survey of Canadian practice. Crit Care Med 2013;41:1745-53.

67. Choong K, Tran N, Clark H. Acute rehabilitation in critically ill children. J Pediatr Intensive Care 2012;1:183-92.

68. Cameron MA, Marsillio LE, Cushman LF, et al. Parents' perspectives on the consent approach for minimal-risk research involving children. IRB 2011;33:6-13.

69. Hough CL. Improving physical function during and after critical care. Curr Opin Crit Care 2013;19:488-95. 
70. Kronick SL, Kurz MC, Lin S, et al. Part 4: Systems of Care and Continuous Quality Improvement: 2015 American Heart Association Guidelines Update for Cardiopulmonary Resuscitation and Emergency Cardiovascular Care. Circulation 2015;132:S397-413.

71. Curtis JR, Cook DJ, Wall RJ, et al. Intensive care unit quality improvement: a "how-to" guide for the interdisciplinary team. Crit Care Med 2006;34:211-8.

72. Gosselink R, Needham D, Hermans G. ICU-based rehabilitation and its appropriate metrics. Curr Opin Crit Care 2012;18:533-9.

73. Herbsman JM, D'Agati M, Klein D, et al. Early Mobilization in the Pediatric Intensive Care Unit: A Quality Improvement Initiative. Pediatr Qual Saf 2020;5:e256.

74. Carrothers KM, Barr J, Spurlock B, et al. Contextual issues influencing implementation and outcomes associated with an integrated approach to managing pain, agitation, and delirium in adult ICUs. Crit Care Med 2013;41:S128-35.

75. Jolley SE, Regan-Baggs J, Dickson RP, et al. Medical intensive care unit clinician attitudes and perceived barriers towards early mobilization of critically ill patients: a crosssectional survey study. BMC Anesthesiol 2014;14:84.

76. Pollack MM, Holubkov R, Glass $\mathrm{P}$, et al. Functional Status Scale: new pediatric outcome measure. Pediatrics

Cite this article as: Morrow BM. Building a culture of early mobilization in the pediatric intensive care unit-a nuts and bolts approach. Transl Pediatr 2021;10(10):2845-2857. doi: $10.21037 /$ tp-20-324 2009;124:e18-28.

77. Pollack MM, Holubkov R, Funai T, et al. Relationship between the functional status scale and the pediatric overall performance category and pediatric cerebral performance category scales. JAMA Pediatr 2014;168:671-6.

78. Corner EJ, Wood H, Englebretsen C, et al. The Chelsea Critical Care Physical Assessment Tool (CPAx): validation of an innovative new tool to measure physical morbidity in the general adult critical care population; an observational proof-of-concept pilot study. Physiotherapy 2013;99:33-41.

79. Ferguson A, Wright S, Stockton K, et al. Abstract PCCLB-39: The Children's Chelsea Critical Care Physical Assessment Tool: Modification of a validated adult assessment tool using Delphi method. Pediatr Crit Care Med 2018;19:255.

80. Morris PE, Goad A, Thompson C, et al. Early intensive care unit mobility therapy in the treatment of acute respiratory failure. Crit Care Med 2008;36:2238-43.

81. Needham DM, Korupolu R, Zanni JM, et al. Early physical medicine and rehabilitation for patients with acute respiratory failure: a quality improvement project. Arch Phys Med Rehabil 2010;91:536-42.

82. Lord RK, Mayhew CR, Korupolu R, et al. ICU early physical rehabilitation programs: financial modeling of cost savings. Crit Care Med 2013;41:717-24. 